\title{
Binding Energies for Electrons of Different Types
}

\author{
Charlotte E. Moore and Henry Norris Russell ${ }^{1}$
}

\begin{abstract}
The binding energy of an electron of given type in any state of a neutral or ionized atom may be defined as that required to remove it along successive terms of a spectral series to its limit. The data regarding electron configurations and limits collected in Atomic Energy Levels, Volumes I and II, permit the calculation of these in a great number of cases.

The present tables give the maximum energy resulting from the addition of an electron of given type (such as $4 p$ ) to the ground states of singly or doubly ionized atoms of the elements $\mathrm{H}$ through $\mathrm{Nb}$; and, when desirable, to one other low state (two in the cases of $\mathrm{Sc}$ and $\mathrm{Y}$ ). These increase smoothly with atomic number except for a remarkable break after a shell of ten $d$-electrons has been half filled.
\end{abstract}

\section{Introduction}

The first extensive table of the binding energy with which an electron of a given type is attached to a neutral or ionized atom in a specified energy level was given by one of us 22 years ago [1]. ${ }^{2}$ It is striking evidence of the extraordinarily rapid advance of spectroscopic theory and analysis during the 5 preceding years that more than 80 percent of the data now available for the same spectra were then included, and that very little change would be required even in the descriptive text. A thorough revision of the data made in connection with the compilation of Bureau Circular 467, Atomic Energy Levels [2], has led as a byproduct to the tables given below.

\section{Definition and Types of Binding Energy}

This binding energy may be defined as that required to remove the electron under consideration from the atom in the specified state without changing any quantized relations except the total quantum number of this electron - thus passing to the limit of the spectral series to which the given energy level belongs.

In the simplest spectra all the series have the same limit. The energy level $L^{\prime}$ (in $\mathrm{cm}^{-1}$ ) corresponding to this limit is given in Circular 467 at the end of the table containing the levels $l^{\prime}$ for the individual states. The binding energy (in $\mathrm{cm}^{-1}$ ) is $L^{\prime}-l^{\prime}$. In electron-volts it is $0.00012395\left(L^{\prime}-l^{\prime}\right)$ [3].

In the case of a spectroscopic term with several components the smallest value of $l^{\prime}$ should be taken, thus giving the maximum binding energy.

In spectra in which terms of two multiplicities arise from the same "running" electron and have the same limit, the smallest value of $l^{\prime}$ tabulated for either of the two should be adopted for $l^{\prime}$, for the same reason.

Thus in $\mathrm{Mg} \mathrm{I}$ [4] the lowest value of $l^{\prime}$ for a $3 p$ electron is 21850 for $3 p{ }^{3} \mathrm{P}_{0}^{\circ}$, that for $3 d$ is 46403 for $3 d^{1} \mathbf{D}_{2}$, while the limit $3 s{ }^{2} \mathrm{~S}_{01 / 2}$ in $\mathrm{Mg}$ II is 61669 . The binding energy is therefore $39819 \mathrm{~cm}^{-1}$, or 4.94 volts, for $3 p$, and $15266 \mathrm{~cm}^{-1}$ or 1.89 volts for $3 d$, as given in table 1.

1 Princeton University Observatory, Princeton, N. J.

2 Figures in brackets indicate the literature references at the end of this paper.

\section{Binding Energy in Complex Spectra}

In the more complex spectra a given electron configuration gives rise to a number of different terms, most of which have three or more components; and the addition of a $p$ or $d$ electron to one of these terms usually produces, in the spectrum of next lower ionization, two triads or pentads of terms of different multiplicity. To reduce the tables to reasonable bulk, only the lowest term belonging to a given configuration in the spectrum of higher ionization is included; and the tabulated binding energy is taken as the difference between the lowest component of this limit and the lowest component (regardless of the multiplicity) of any member of triads or pentads of terms arising from it. It thus represents the maximum value for an electron of the given type. The designation of each term that is included in the tables is given as it appears in Volume I or II of Circular 467 [2].

\section{Cases With Two Different Limits}

In certain important spectra, however, two or occasionally three, electron configurations give terms at nearly the same level, as in the classical example of the group $\mathrm{Ca}$ II to $\mathrm{Cu}$ II where the lowest configuration comes sometimes from $3 d^{n-1}$ and in other cases from $3 d^{n-2} 4 s$. In such cases the lowest term arising from each of these configurations is adopted as a tabular limit, and the binding energies from $\mathrm{Ca}$ I to $\mathrm{Cu}$ I corresponding to the addition of an electron of given type to each of them are given in table 1. Those with limit $3 d^{n-1}$ are distinguished by " $\mathrm{A}$ " at the head of the column, and the others by "B." Only terms of group A appear in K I and of type B in $\mathrm{Zn}$ I. A similar situation, involving $4 d$ and $5 s$ electrons appears from $\mathrm{Rb}$ I to $\mathrm{Cd}$. A dash in the table indicates that no such term exists.

For doubly ionized atoms a similar situation rarely arises. From Ca III to $\mathrm{Cu}$ III, for example, the lowest level always comes from $3 d^{n-2}$. The addition of $3 d$ or $4 s$ to this level gives the terms in Ca II to $\mathrm{Cu}$ II thus designated in table 2 , which serve as the limits for groups $\mathrm{A}$ and $\mathrm{B}$ from $\mathrm{Ca}$ I to $\mathrm{Cu}$.

The further addition of $4 s$ or $3 d$, respectively, gives the same term in each arc spectrum - namely, 
TABLE 1.-Maximum binding energy of an electron-neutral atoms

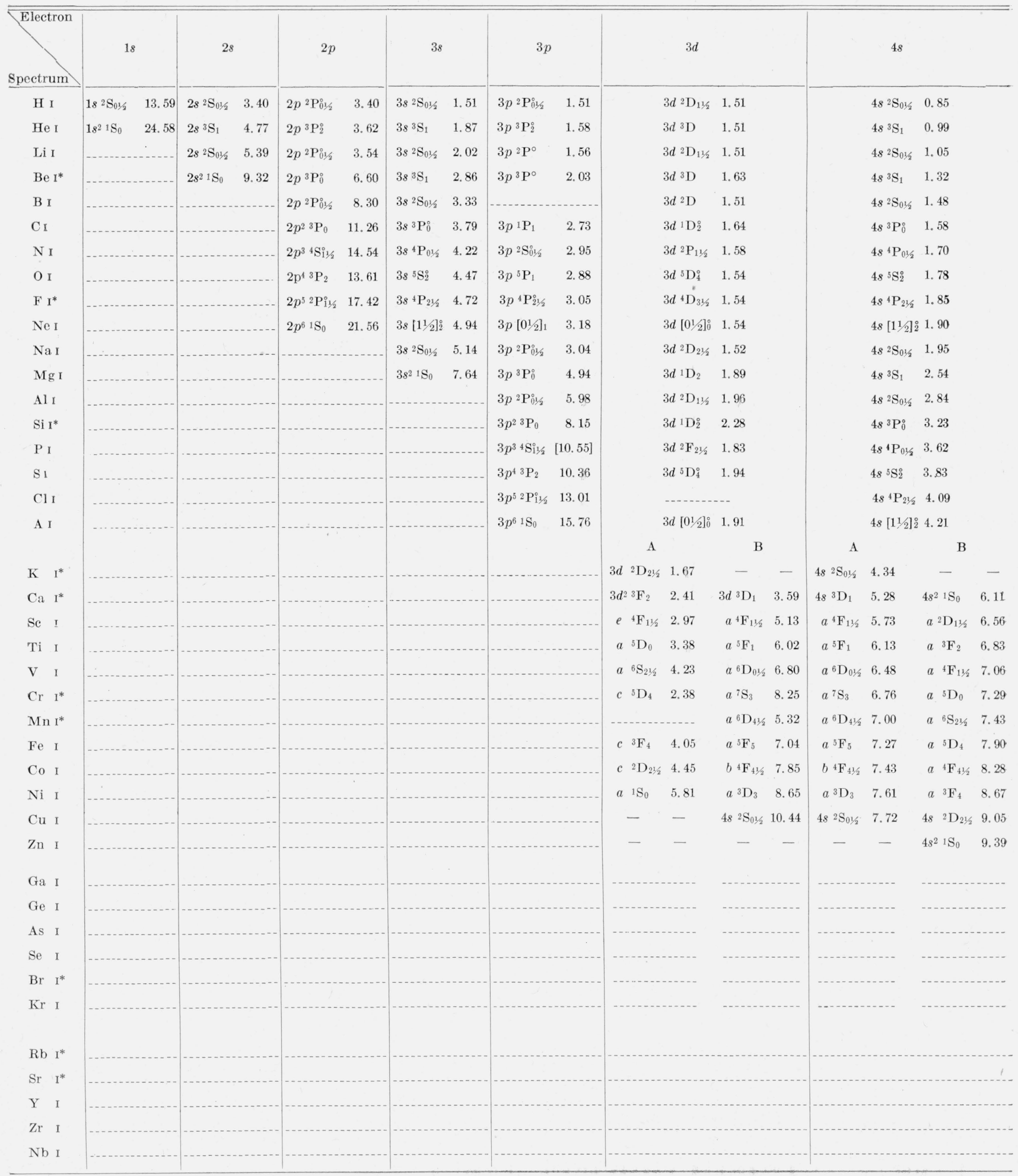

*An asterisk refers to the special notes on individual spectra. 
TABLE 1.-Maximum binding energy of an electron-neutral atoms-Continued

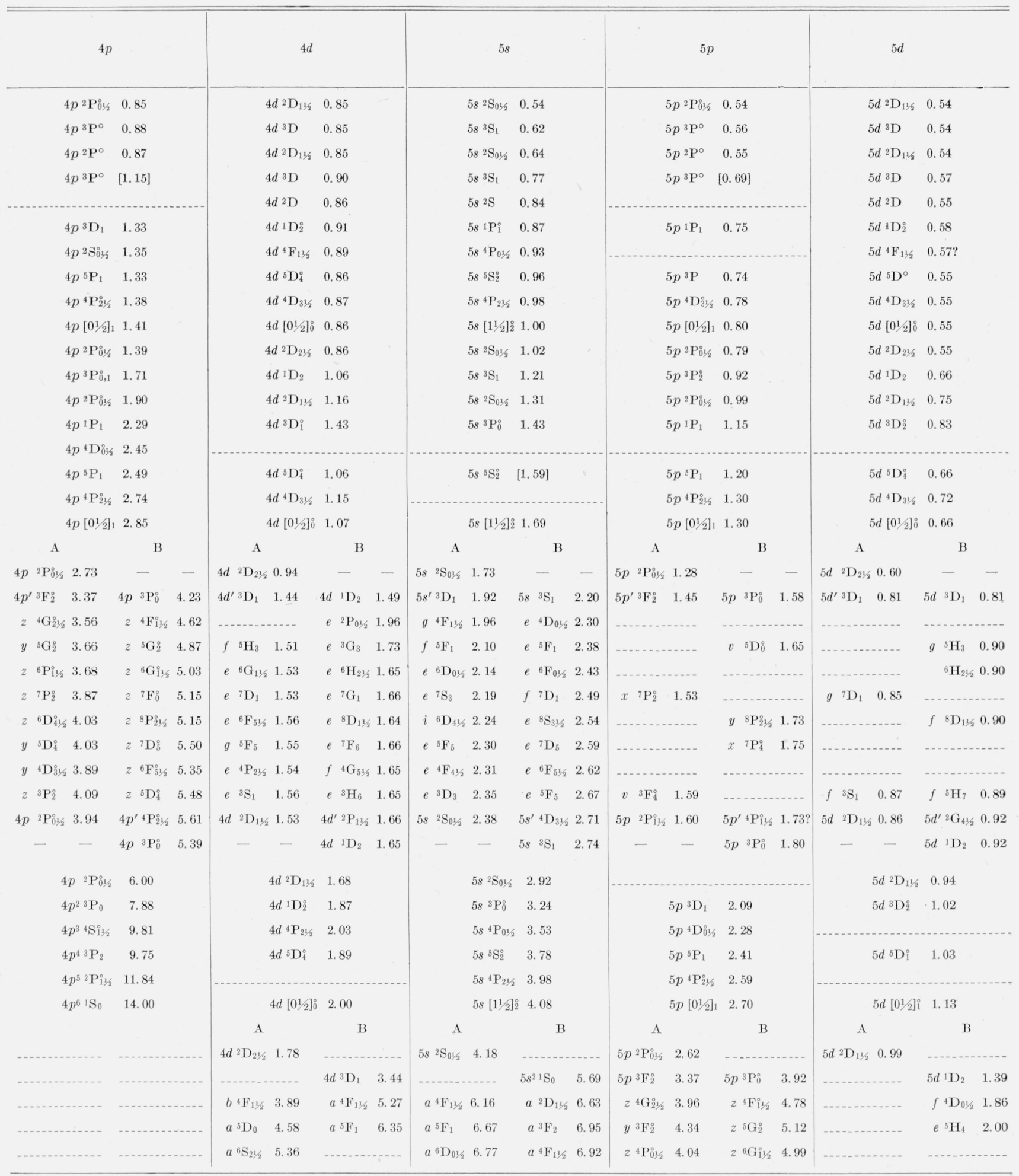


TABLE 2. Maximum binding energy of an electron-singly ionized atoms

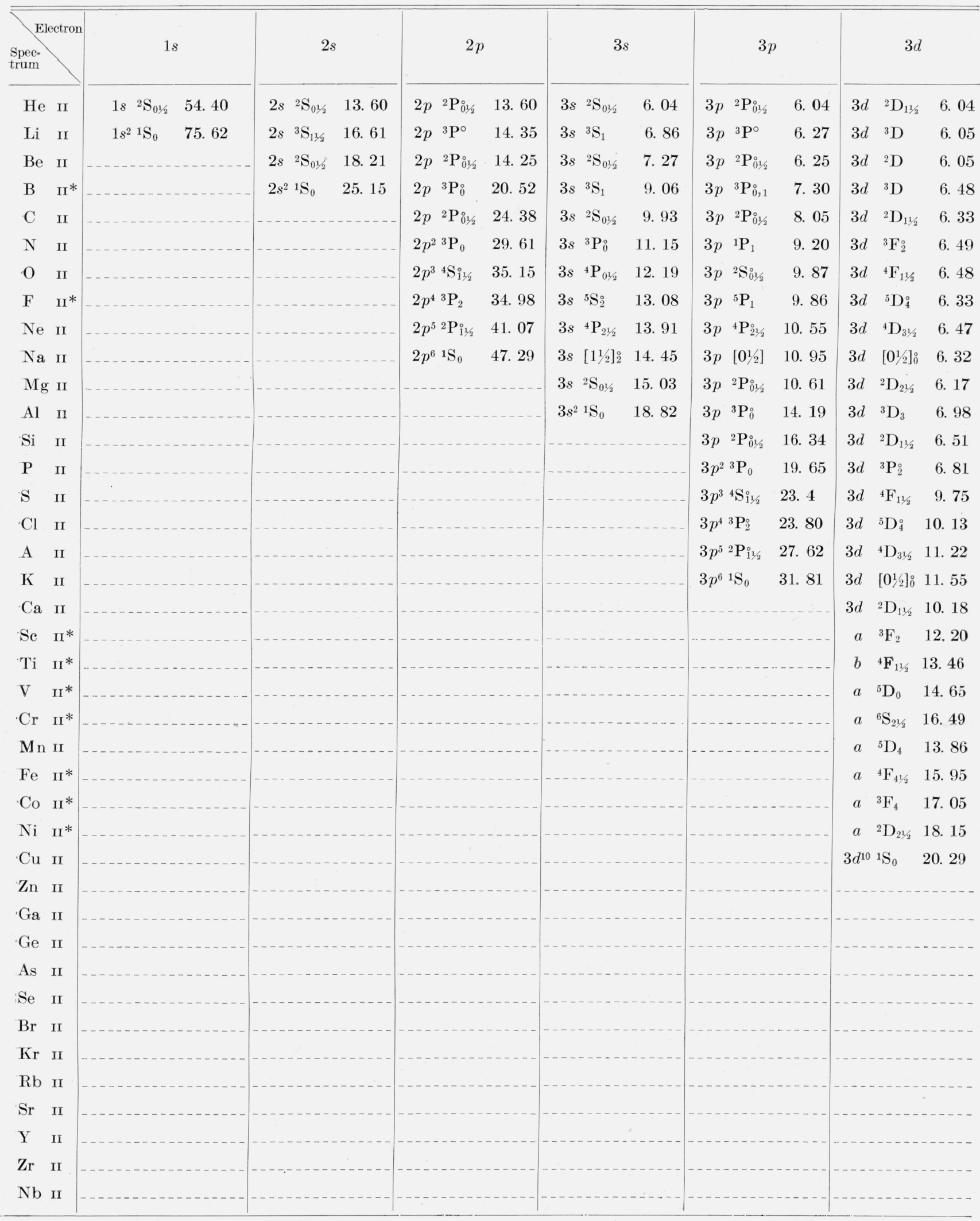

${ }^{*}$ An asterisk refers to special notes on individual spectra. 
TABLE 2.-Maximum binding energy of an electron-singly ionized atoms - Continued

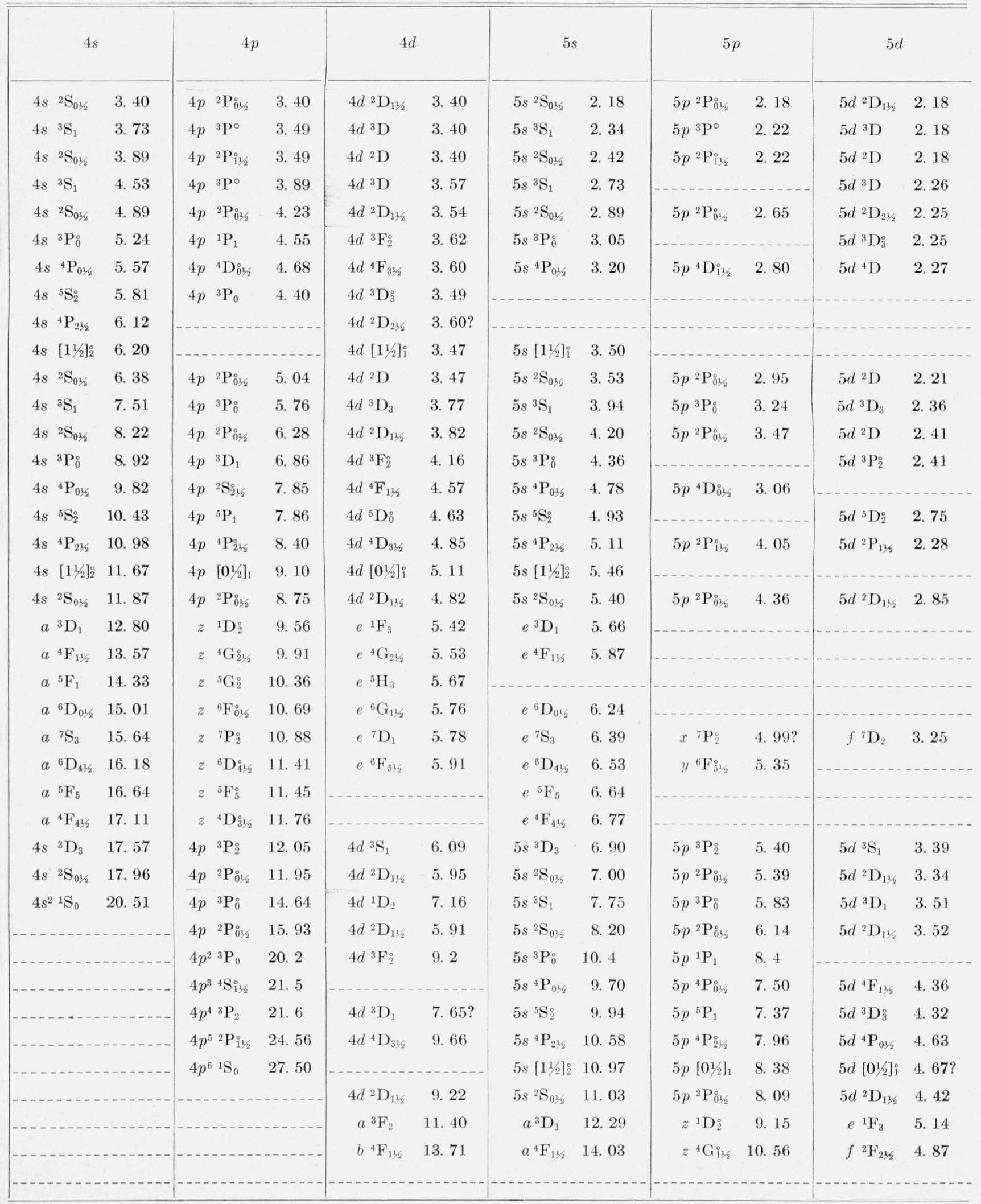


the lowest belonging to the configuration $3 d^{n-1} 4 s$. The differences in the binding energies given for $3 d$ and $4 s$ in table 2 and for $3 d(\mathrm{~B})$ and $4 s(\mathrm{~A})$ in table 1 are therefore identical, barring occasional effects of rounding off the separate values.

\section{Numerical Example}

As an example of calculation in this case, take V I [5]. Here the limit of group $\mathrm{A}$ is $3 d^{4} a^{5} \mathrm{D}_{0}$ in V II at a level of $54361 \mathrm{~cm}^{-1}$ above the ground level of $\mathrm{VI}$, while that of group B is $3 d^{3} 4 s a^{5} \mathrm{~F}_{1}$, which lies higher by $2605 \mathrm{~cm}^{-1}$, or at 56966 . We then find for each added electron the configuration, term, and lowest level given below:

\begin{tabular}{|c|c|c|c|c|c|c|c|c|}
\hline \multirow{2}{*}{$\begin{array}{l}\text { Added } \\
\text { electron }\end{array}$} & \multicolumn{4}{|c|}{ Group A } & \multicolumn{4}{|c|}{ Group B } \\
\hline & Config. & Term & Level & $\begin{array}{c}\text { Binding } \\
\text { energy }\end{array}$ & Config. & Term & Level & $\begin{array}{l}\text { Binding } \\
\text { energy }\end{array}$ \\
\hline $3 d$ & $3 d^{5}$ & $a^{6} \mathrm{~S}_{21 / 2}$ & 20202 & $\begin{array}{c}e v \\
4.23\end{array}$ & $3 d^{4} 48$ & $a^{6} \mathrm{D}_{01 / 2}$ & 2112 & $\begin{array}{c}e v \\
6.80\end{array}$ \\
\hline 48 & $3 d^{4} 4 s$ & $a^{6} \mathrm{D}_{01 / 3}$ & 2112 & 6.48 & $3 d^{3} 4 s^{2}$ & $a^{4} \mathrm{~F}_{11 / 2}$ & 0 & 7. 06 \\
\hline $4 p$ & $3 d^{4} 4 p$ & $z^{6} \mathrm{P}$ & 24628 & 3.68 & $3 d^{3} 4 s 4 p$ & $z^{6} \mathrm{G}^{\circ} 1 / 2$ & 16361 & 5. 03 \\
\hline
\end{tabular}

The configuration for each case is first worked out; the lowest term and level for this is found by inspection of the tables in Circular 467. The difference between this level and the appropriate limit, multiplied by $1.2395 \times 10^{-4}$ then gives the binding energy.

It is worth noting that, in this case, none of the binding energies given above corresponds to the principal ionization potential of the atom. This is given by the transition $3 d^{3} 4 s^{2} a{ }^{4} \mathrm{~F}_{1 / 2}$ to $3 d^{4} a{ }^{5} \mathrm{D}_{0}$ and involves a double electron jump. Such cases are rare, the only others in the present work occur in Co I, where the transition is from $3 d^{7} 4 s^{2} a^{4} \mathrm{~F}_{41 / 5}$ to $3 d^{8} a^{3} \mathrm{~F}_{4}$, and in NiI where it is from $3 d^{8} 4 s^{2} a^{3} \mathrm{~F}_{4}$ to $3 d^{9} a^{2} \mathrm{D}_{235}$.

\section{Case of Scandium and Yttrium}

Theoretically, the spectra of singly ionized atoms from Sc II to Zn II should contain many terms with limits in the third spectra arising from configurations $3 d^{n-3} 4 s$, which should be related to those with limits from $3 d^{n-2}$ exactly as group B in the first spectra is related to group A. The corresponding limits are, however, so high that scarcely any such terms have been observed except in scandium, and in yttrium in the next long period. It, therefore, appears admissible to give the data for the few known terms of this type separately in table 3.

In this table the first four columns (which have no counterparts in tables 1 and 2) give data regarding the limits here involved - the "level" being measured from that in which one $d$ electron is substituted for an $s$-that is, the limit adopted in table 1, group B, for first spectra and in table 2 for second spectra.

The remaining part of the table is arranged exactly as in tables 1 and 2. The entries for $3 d$ in Sc I and $4 d$ in $\mathrm{Y}_{\mathrm{I}}$ exceed those for $4 s(\mathrm{~B})$ and $5 s(\mathrm{~B})$ by the level difference in the fourth column. The remaining energy levels appear only in table 3 .
The new limiting configuration in $\mathrm{Y}_{\mathrm{II}}$ is the lowest in the spectrum, and the transition from this to the lowest level in Y I, $5 s^{2} 4 d^{2} \mathrm{D}$, gives the principal ionization potential 6.53 volts, which does not appear in table 1, though it corresponds to a single jump of a $4 d$ electron.

\section{Description of the Tables}

Table 1 gives the binding energies of $n s, n p$, and $n d$ electrons for neutral atoms of the elements $\mathrm{H}$ to $\mathrm{Nb}$, and for values of $n$ from 1 to 5 , excluding those belonging to complete shells. Table 2 gives similar data for singly ionized atoms from $\mathrm{He}$ II to $\mathrm{Nb}$ II. Full explanations of their significances, of the groups $A$ and $B$ given for first spectra, and of the way in which they have been calculated, are given in sections 2 to 5 . Table 3 contains certain additional values that need be recorded only for scandium and y trium. (See section 6.)

Most of the vacant spaces in the tables correspond to energy levels that are so situated that the strongest lines corresponding to transitions involving them lie in the infrared or far in the ultraviolet and have not yet been observed.

In a number of cases some terms belonging to the electron configurations in question are known, but there is no doubt that the lowest level of the configuration has not yet been found (usually for the reason just mentioned). Here again the entry is left blank (or an estimated value is occasionally given in brackets).

These, and various other instances in which comments are required, are discussed in the notes following the tables. Full discussions are also given of a very few cases in which it now appears that the electron configurations assigned in AEL should be changed.

The order in which the electron types are presented in tables 1 and 2 is that in which they form "complete shells" and enter the inner part of the atomic structure - as is shown by the termination of the vertical column for each.

Until the given electron is involved in the production of such a shell, the increase of its binding energy with atomic number is slow, with occasional irregularities arising from the choice of the lowest level in each configuration rather than the mean. During the formation of the shell involving the given electron, the increase in binding energy is much faster. The remarkable reversal of this increase at the point where a shell of $d$ electrons is half filled may be tentatively explained as an effect of the reversal of the spin of the sixth added electron demanded by Pauli's principle.

\section{References}

[1] H. N. Russell, Astroph. J. 70, 11 (1929).

[2] C. E. Moore, NBS Circular 467 I, (1949); II (1952).

[3] R. T. Birge, Rev. Mod. Phys. 13, No. 4, 233 (1941).

[4] Atomic Energy Levels, NBS Circular 467 I, 107 (1949).

[5] Atomic Energy Levels, NBS Circular 467 I, 292 to 298 (1949).

[6] K. Lidén, Ark. Fysik. 1, No. 9, 259 (1949). 
TABLE 3. Additional binding energies for Sc and $\mathrm{Y}$

\begin{tabular}{|c|c|c|c|c|c|c|c|c|c|c|c|c|c|}
\hline \multicolumn{4}{|c|}{ Limit } & \multicolumn{10}{|c|}{ Binding Energy } \\
\hline Spectrum & Config. & Desig. & Level & Spectrum & $3 d$ & 48 & $4 p$ & & $4 d$ & $5 s$ & $5 p$ & $5 d$ & \\
\hline $\mathrm{Se}$ II & $4 s^{2}$ & $a^{1} \mathrm{~S}_{0}$ & $\begin{array}{l}e v \\
1.45\end{array}$ & Se I* & $a^{2} \mathrm{D}_{11 / 2} \quad 8.01$ & & $x{ }^{2} \mathrm{P}_{01 / 3}^{\circ}$ & 4. 22 & & & & & \\
\hline Y II & $5 s^{2}$ & $a^{1} \mathrm{~S}_{0}$ & -0.10 & Y I & & & & & $a^{2} \mathrm{D}_{11 / 2} \quad 6.53$ & & $z^{2} \mathrm{P}_{01 / 3}^{\circ} \quad 5.22$ & $g^{2} \mathrm{D}_{11 / 2}$ & 1.24 \\
\hline Se шI & $4 s$ & $4 s \cdot{ }^{2} \mathrm{~S}_{01 / 2}$ & 3.17 & Se II & $a{ }^{3} \mathrm{D}_{1} \quad 15.96$ & $a^{1} \mathrm{~S}_{0} \quad 14.51$ & $y{ }^{3} \mathrm{P}_{0}^{\circ}$ & 11.13 & & & & & \\
\hline Y III & $5 s$ & $5 s{ }^{2} \mathrm{~S}_{01 / 2}$ & 0.93 & Y II & & & & & $\begin{array}{ll}a^{3} \mathrm{D}_{1} & 13.22\end{array}$ & $\begin{array}{ll}a^{1} \mathrm{~S}_{0} & 13.32\end{array}$ & $z^{3} \mathrm{P}_{0}^{\circ} \quad 10.41$ & $g 1 \mathrm{D}_{2}$ & 5.57 \\
\hline
\end{tabular}

${ }^{*}$ An asterisk refers to special notes on individual spectra.

Binding Energies-Notes to Tables 1, 2, and 3

[An asterisk $(*)$ in the tables applies to the following spectra]

Be I Intersystem combinations between the singlet and triplet terms have not been observed. The relative positions of the two sets of terms are excellently determined by long series, the relative uncertainty being about $\pm 2 \mathrm{~cm}^{-1}$.

The terms $4,5 p^{3} \mathrm{P}^{\circ}$ have been calculated from the series formula but not confirmed by observation. They are entered in brackets in the table.

F I See reference [6]. Only one level is known for $5 p$, perhaps not the lowest.

Si I The general run of binding energies for the $3 d$ electron indicates that the configurations of the two lowest ${ }^{3} \mathrm{D}^{\circ}$ terms as given in Atomic Energy Levels, I, p. 145 , should be interchanged to read as follows:

\begin{tabular}{|l|c|c|}
\hline Configuration & Designation & Level \\
\hline $3 s 3 p^{3}$ & $3 p^{3}{ }^{3} \mathrm{D}_{\mathrm{i}}^{\circ}$ & 45276.20 etc. \\
$3 s^{2} 3 p\left({ }^{2} \mathrm{P}^{\circ}\right) 3 d$ & $3 d{ }^{3} \mathrm{D}_{\mathrm{i}}^{\circ}$ & 48399.15 etc. \\
\hline
\end{tabular}

If this change is made, the binding energy for $3 d$ is then determined from the level $3 d^{1} \mathrm{D}_{2}^{\circ}=47351.50$, as has been done here. This change should be confirmed by further observations.

$\mathrm{P}_{\mathrm{I}}$ The ionization potential estimated by W. Finkelnburg and F. Stern, (Phys. Rev. $7 \%, 303$ L (1950)), from the regularities in the run of the screening constants is preferable to that given in $[2$, page 163$]$. The corresponding limit $85115 \mathrm{~cm}^{-1}$ has been used in calculating the binding energies.

The term $5 s^{4} \mathrm{P}$ should be rejected.

K I-Zn I Group A: The limit is the lowest level of the configuration $3 d^{n-1}$ in the singly ionized atom $[n=1(\mathrm{~K})$ to $11(\mathrm{Cu})]$.

Group B: The limit is the lowest level of the configuration $3 d^{n-2} 4 s$ in the singly ionized atom $[n=2(\mathrm{Ca})$ to $12(\mathrm{Zn})]$.

Ca I The following term has recently been reported by C. J. Humphreys (J. Research NBS 47, 262 (1951) RP2252).

\begin{tabular}{|c|c|c|c|}
\hline Designation & $J$ & Level & Interval \\
\hline $3 d^{2}{ }^{3} \mathrm{~F}$ & $\begin{array}{l}2 \\
3 \\
4\end{array}$ & $\begin{array}{l}43474.87 \\
43489.19 \\
43508.10\end{array}$ & $\begin{array}{l}\text { 14. } 32 \\
\text { 18. } 91\end{array}$ \\
\hline
\end{tabular}

It has been used to calculate the binding energy for $3 d$, group $\mathrm{A}$ in the table.

Sc I Table 3. Racah has suggested that the configuration of $x^{2} \mathrm{P}^{\circ}$ should be corrected in "AEL" to read $4 s^{2}\left(a^{1} \mathbf{S}\right) 4 p$ instead of $3 d^{2}\left(a^{1} \mathbf{S}\right) 4 p$. Assuming this correction, the binding energy for $4 p$ comes from this term.

Cr I The binding energy for $5 p$, group $\mathrm{B}$, is omitted because the general run of values in this column indicates that the lowest known level $\left(s^{5} \mathrm{~F}_{i}^{\circ}\right)$ is not the lowest in the triads to be expected, and does not, therefore, give the maximum binding energy.

Mn I The lowest level for $3 d$, group $\mathrm{A}$, is not known in Mn I. The level $c{ }^{4} \mathrm{P}_{21 / 2} 51638.17$ is not the lowest, and has, therefore, not been used.

Br I The magnitudes of the binding energies indicate that the terms from the $4 d$ and $5 d$ electrons have not been found, and that the known nd terms are from $6 d$ and $7 d$.

Rb I-Nb I Group A: The limit is the lowest level of the configuration $4 d^{n-1}$ in the singly ionized atom $[n=1(\mathrm{Rb})$ to $5(\mathrm{Nb})]$.

Group B: The limit is the lowest level of the configuration $4 d^{n-2} 5 \mathrm{~s}$ in the singly ionized atom $[n=2(\mathrm{Sr})$ to $5(\mathrm{Nb})]$.

Sr I The known term $4 d^{2}{ }^{3} \mathrm{P}$ from this configuration is undoubtedly higher than the unknown $4 d^{2}{ }^{3} \mathrm{~F}$ term. Consequently no entry is given in table 1 for $4 d$, group A.

B II No intersystem combinations connecting the singlet and triplet systems of terms have been observed. Since the relative positions of the two sets of terms have been estimated, all entries in the table except that for $2 s$ are subject to an uncertainty that probably does not exceed \pm 0.03 volt.

F II The quintet terms are not connected with the rest by observed intersystem combinations. Since their relative positions have been estimated, the tabular entries for $3 s, 3 p, 3 d$, and $4 s$ are subject to an uncertainty probably not exceeding \pm 0.03 volt.

Se II

Ti II

V II Revised limit given by H. N. Russell (J. Opt. Soc. Am.

Cr II 40,619 (1950)) used for the calculation of binding Fe II energies.

Co II

$\mathrm{Ni}$ II 